\title{
Extension of $193 \mathrm{~nm}$ Lithography by Chemical Shrink Process
}

\author{
Takashi Sekito, Yuriko Matsuura, and Tatsuro Nagahara \\ Merck Performance Materials Manufacturing G.K. \\ Global R\&D Lithography, IC Materials, \\ 3330 Chihama, Kakegawa, Shizuoka, 437-1412, Japan
}

\begin{abstract}
Roughness improvement of Negative Tone Development (NTD) photoresist by chemical shrink process is discussed in this paper for the extension of $193 \mathrm{~nm}$ lithography. Addition to feature size shrinkage, improvement of photoresist roughness is also important subject for advanced node device manufacturing. New shrink materials were evaluated by Scan Probe Microscope (SPM) and a material improved surface roughness by $37 \%$. Moreover, $28 \%$ improvement of Contact Edge Roughness (CER) was confirmed.
\end{abstract}

Keywords: shrink, NTD, photoresist, CER, CDU, roughness, ArF, AFM, SPM, polymer aggregate

\section{Introduction}

It is very important to study extension of $193 \mathrm{~nm}$ lithography until EUV lithography can launch in high volume manufacturing phase. In such situations, NTD process was developed to achieve finer patterning than standard positive tone lithography, especially for trench and hole features patterned using dark mask. This NTD process is a negative tone imaging by development with organic solvent such as n-butyl acetate $[1,2]$. However, the pattern size that is achieved by NTD is not sufficient for advanced node devices, so that additional techniques to attain further fine patterning are being sought.

Chemical shrink is a well-known process which gives simple process with low $\mathrm{CoO}$ (cost of ownership) to fabricate small contact hole and narrow trench patterns [3]. Based on these advantages, chemical shrink materials have been widely used in semiconductor device production. Of course this chemical shrink process can be combined with NTD process, and its achievement of fine patterning was reported previously [4,5].

For the extension of $193 \mathrm{~nm}$ lithography, addition to pattern size shrinkage, pattern roughness control is also important subject [6].

In this paper, the possibility to improve pattern roughness and pattern shrinkage simultaneously by chemical shrink materials is studied. For this study, SPM and Scan Electron Microscope (SEM) were utilized.

\section{Experimental}

2.1. Chemical shrink process

For the evaluation of shrink materials, NTD photoresist pattern of $65 \mathrm{~nm}$ Contact Hole $(\mathrm{CH})$ was prepared.

Three kinds of chemical shrink materials were formulated as shown in Table 1. The main components of shrink materials are polymer, additive and De-Ionized (DI) water. Shrink process flow is shown in Figure 1. Prepared shrink materials were spin-coated on NTD photoresist pattern. Then, the samples were baked at $130{ }^{\circ} \mathrm{C}$ for $60 \mathrm{sec}$. Finally, they were developed by D. I. Water for $60 \mathrm{sec}$.

\subsection{Measurement}

The surface roughness and film thickness of photoresist pattern was measured using Hitachi High-Tech Science Scan Probe Micro- 

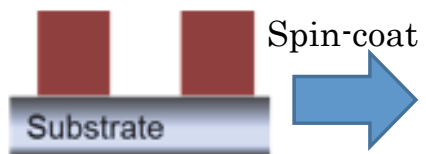
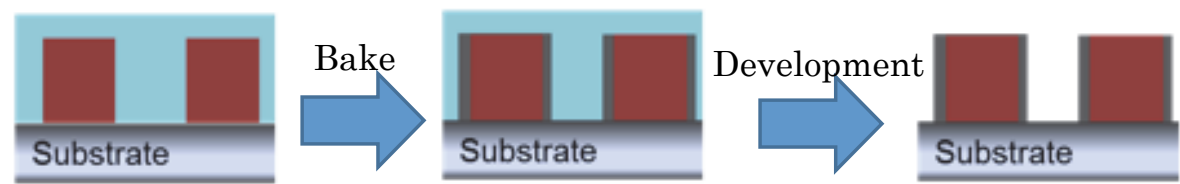

Fig. 1. Shrink process flow.

Table 1. Shrink material comportment.

\begin{tabular}{clll}
\hline Component & NSM-A & NSM-B & NSM-C \\
\hline Polymer & Polymer-A & Polymer-A & Polymer-A \\
\hline Additive & Additive-A & Additive-B & Additive-C \\
\hline Solvent & D. I. Water & D. I. Water & D. I. Water \\
\hline
\end{tabular}

scope (SPM) AFM5300E with a microtip, Olympus OMCL-AC240TS-R3. The shape of micro-tip is conical whose radius of apex is $7 \mathrm{~nm}$. The microtip is sharp enough to measure film thickness at $\mathrm{CHs}$. Sample Intelligent Scan Dynamic Force Mode (SIS-DFM) was used for measurement mode of SPM [7]. It is said that SIS-DFM is a suitable measurement mode to avoid microtip damage with textured sample compared to normal DFM.

CD, Local CD Uniformity (L-CDU) and CER were measured using Hitachi RS5500 with off-line software Offline CD Measurement Software v.6.00.

\section{Results and Discussion}

3.1. Surface roughness

SPM topographic image of NTD photoresist with or without shrink process is shown in Figure 2. In the ADI (After Development Inspection) image, the surface was covered grains of $5 \sim 20 \mathrm{~nm}$. The grains could be considered polymer aggregates of photoresist [8,9]. Grain sizes in ASI (After Shrink Inspection) image with NSM-A and B were smaller than that of ADI. In image of ASI with NSM-C, few grain of small size was seen.

SPM phase image of ADI and ASI is shown in Figure 3. Many grains (grain size: 5 20 $\mathrm{nm})$ were also found in the image of ADI especially around CHs. Grain size of ASI image is much smaller than that of ADI image in phase mode as well as topographic mode. While CHs size of ASI in phase image looks much smaller than that of ADI, this may be caused by increase of interaction between SPM microtip and sample surface. For example, softening of surface, electrostatic charging, absorption of water are the possible causes.

Photoresist film thickness and surface roughness of photoresist pattern with and without shrink process is shown in Table 2. All of the thickness are around 50nm, there are no thickness loss and increase by shrink process.

Root-mean-square roughness (Rrms) of ASI is smaller than that of ADI. Rrms of ASI with NSM-C is the smallest among all the samples. Mechanism of surface smoothing will be studied further in future.
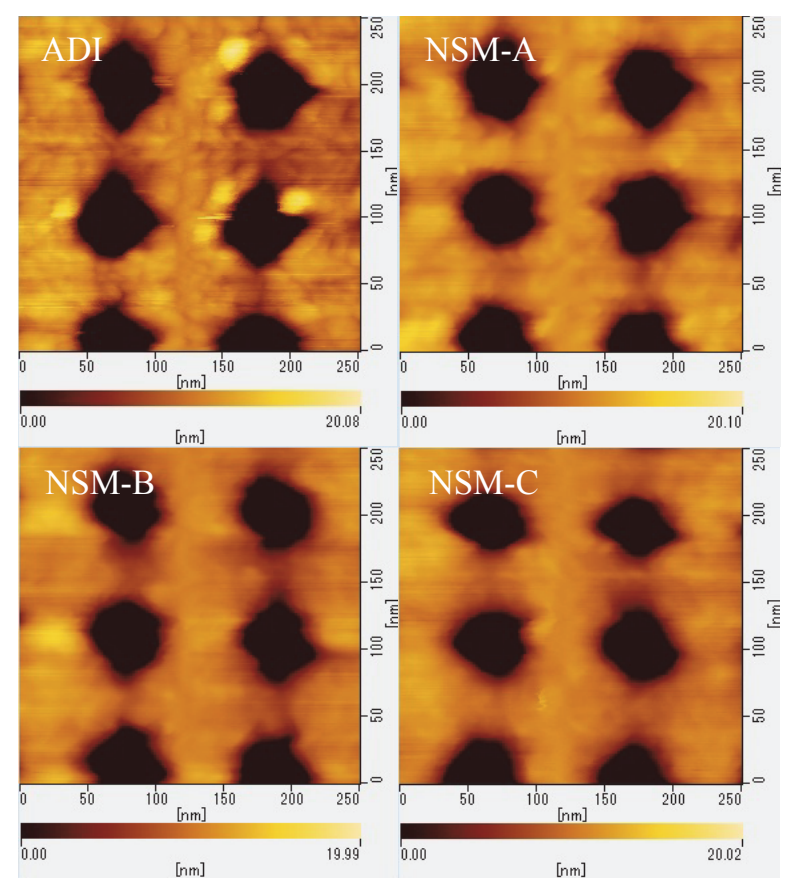

Fig. 2. SPM topographic image of ADI and ASI using NSM-A, B and C. 

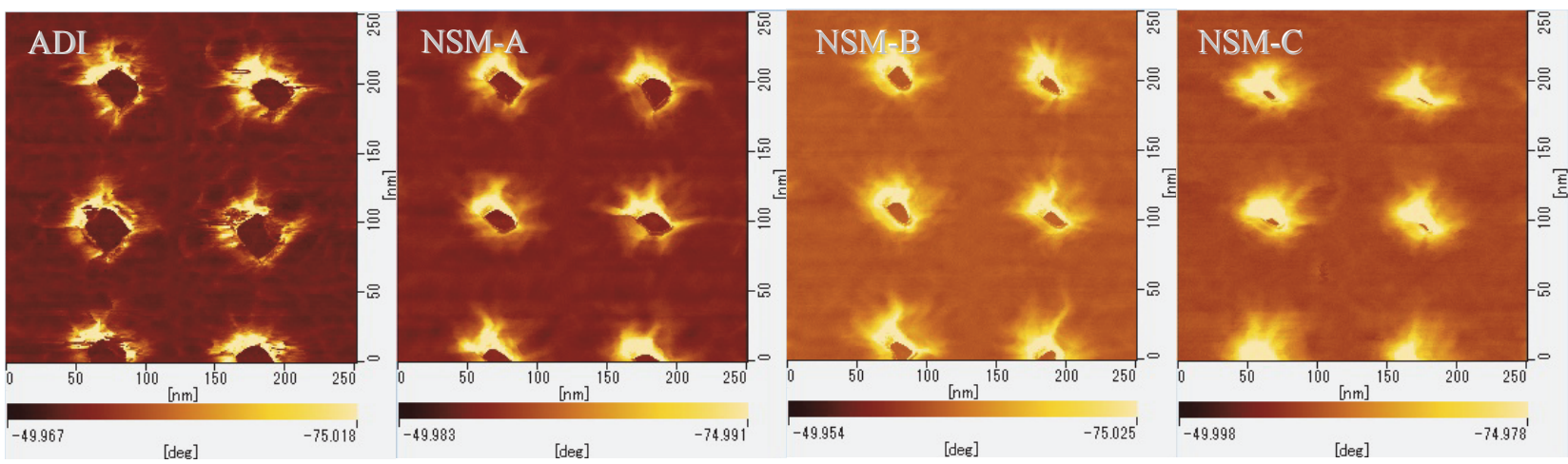

Fig. 3. SPM phase image of ADI and ASI using NSM-A, B and C.

Table 2. Surface roughness and photoresist pattern thickness of ADI and ASI of NSM-A, B and C.

\begin{tabular}{ccccc}
\hline Sample & ADI & NSM-A & NSM-B & NSM-C \\
\hline Photoresist pattern thickness (nm) & 50.27 & 51.17 & 50.24 & 50.61 \\
\hline Surface roughness $* \mathrm{R}_{\text {rms }}(\mathrm{nm})$ & 12.38 & 10.16 & 9.41 & 7.83 \\
\hline$* *$ Improvement ratio of $\mathrm{R}_{\text {rms }}$ from ADI & - & $18 \%$ & $24 \%$ & $37 \%$ \\
${ }^{*} R_{\text {rms }}=\sqrt{\frac{1}{N} \sum_{n-1}^{N} R_{n}{ }^{2}}{ }^{* *}$ Improvement ratio $=\frac{R_{\text {rcm } \text { ASI }}}{R_{\text {rms ADI }}-R_{\text {rcm ASI }}}$
\end{tabular}

Table 3. CER and L-CDU of ADI and ASI of NSM-A, B and C.

\begin{tabular}{|c|c|c|c|c|}
\hline Sample & ADI & NSM-A & NSM-B & NSM-C \\
\hline Shrinkage $(\mathrm{nm})$ & - & 3.9 & 11.3 & 16.4 \\
\hline $\operatorname{CER}(3 \sigma, \mathrm{nm})$ & 2.5 & 2.4 & 2.1 & 1.8 \\
\hline Improvement ratio of CER & - & $4 \%$ & $16 \%$ & $28 \%$ \\
\hline L-CDU $(3 \sigma, n m)$ & 4.5 & 4.1 & 3.9 & 3.3 \\
\hline Improvement ratio of L-CDU & - & $9 \%$ & $13 \%$ & $20 \%$ \\
\hline SEM image & 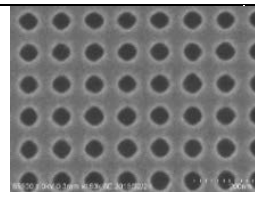 & 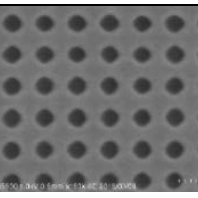 & $\because \because \because \vdots:$ & $\begin{array}{l}: \cdots \\
\therefore\end{array}: \cdots$ \\
\hline
\end{tabular}

\subsection{CER and L-CDU}

CER and L-CDU of $\mathrm{CHs}$ with and without shrink process with NSM-A, B and- C are shown in Table 3. CERs and L-CDUs of ASIs were smaller than that of ADI. Furthermore, both CER and L-CDU of NSM-C showed the best improvement among tested samples. The relationship between surface roughness improvement and CER improvement will be studied further.

\section{Conclusion}

We conducted a study to extend $193 \mathrm{~nm}$ lithography with chemical shrink materials. Especially, we focused on roughness improvement addition to fundamental shrink function. Effect of additives were discussed and finally it was found that NSM-C achieved the highest shrinkage and the best roughness improvement.

\section{References}

1. S. Tarutani, H. Tsubaki, and S. Kanna, Proc. SPIE, 6923 (2008) 69230F.

2. S. Tarurani, S. Kamimura, K. Fujii, K. Katou and Y. Enomoto, Proc. SPIE, 7972 (2011) $79720 \mathrm{~N}$

3. T. M. Terai, T. Kumada, T. Ishibashi, T. Hanawa, N. Satake, Y. Takano, Proc. SPIE, 6153 (2006) 61532I.

4. Y. Miyamoto, J. Sagan, M. Padmanaban, G. Pawlowski and T. Nagahara, Proc. SPIE, 9051 (2014) 905123.

5. Y. Miyamoto, T. Sekito, J. Sagan, Y. 
Horiba, T. Kinuta, T. Nagahara and S. Tarutani, Proc. SPIE, $9425 \quad$ (2015) 942520.

6. S. Xiong, J. Bokor, Q. Xiang, P. Fisher, I. Dudley, and $\mathrm{P}$ Rao, Proc. SPIE, 4689 (2002) 733.

7. M. Yasutake, K. Watanabe, S. Wakiyama and T. Yamaoka, Jpn. J. Appl. Phys., 45 (2006) 1970.

8. A. Kawai and D. Inoue, J. Photopolym. Sci. Technol., 15 (2002) 127.

9. A. Kawai, J. Photopolym. Sci. Technol., 16 (2003) 381. 